\title{
Reduction of Hip2 suppresses gastric cancer cell proliferation, migration, invasion and tumorigenesis
}

\author{
Jugang $\mathbf{W u}^{\#}$, Baoxing Tian\#, Jianjun Yang, Haizhong Huo, Zhicheng Song, Jiwei Yu, Yan Gu \\ Department of General Surgery, Shanghai Ninth People's Hospital, Shanghai Jiao Tong University School of Medicine, Shanghai 200011, China \\ Contributions: (I) Conception and design: J Wu; (II) Administrative support: Y Gu; (III) Provision of study materials or patients: Y Gu, J Yu; (IV) \\ Collection and assembly of data: J Wu, B Tian; (V) Data analysis and interpretation: J Wu; (VI) Manuscript writing: All authors; (VII) Final approval \\ of manuscript: All authors. \\ \#These authors contributed equally to this work. \\ Correspondence to: Prof. Yan Gu. Department of General Surgery, Shanghai Ninth People's Hospital, Shanghai Jiao Tong University School of \\ Medicine, 639, Zhizaoju Road, Shanghai 200011, China. Email: yangu@sjtu.edu.cn.
}

Background: Hip2, a ubiquitin-conjugating enzyme, has been shown to modulate the stability of cyclin B1, a cell cycle regulator. However, the function of Hip2 in gastric cancer (GC) remains largely elusive.

Methods: The expression of Hip2 in GC cell lines was analyzed by RT-qPCR, Western Blotting and Immunohistochemical Staining. shRNA was utilized to knock down the expression of Hip2. Cell growth, cell cycle, migration, invasion and tumorigenesis were performed by CCK-8, BrdU staining, flow cytometry, wound healing, transwell migration and invasion, and xenograft assay, respectively.

Results: Hip2 was highly expressed in GC cell lines and patients. High level of Hip2 indicated poor prognosis. Knockdown of Hip2 suppressed cell growth, lead to G2/M phase arrest, and reduced cell migration and invasion in vitro. Furthermore, downregulation of Hip2 inhibited tumorigenesis in vivo.

Conclusions: Elevated expression of HIP2 in GC patients suggested poor prognosis. Reduction of Hip2 suppressed GC progression, indicating that Hip2 may be a potential target for the management of GC.

Keywords: Hip2; gastric cancer (GC); proliferation; migration and invasion; tumorigenesis

Submitted Aug 15, 2019. Accepted for publication Nov 15, 2019.

doi: $10.21037 /$ tcr.2019.12.12

View this article at: http://dx.doi.org/10.21037/tcr.2019.12.12

\section{Introduction}

Gastric cancer (GC) is the sixth most common malignancy and the second leading cause of cancer-related death worldwide (1). In China, the new cases of GC were 679,100 and the GC-related deaths were 498,000 in 2015 (2). In comparison to females (new cases 477,700 in 2015), males (new cases 201,400 in 2015) are much more vulnerable to GC (2). The high capability of invasion and metastasis in GC patients largely accounts for the poor prognosis. However, there is currently no effective method for the successful treatment of progressive or metastatic GC. Therefore, finding a protein that regulates cell apoptosis, growth arrest, and the migration and invasion behavior of GC cells is a prerequisite for the development of new therapies.

Hip2 (short for huntingtin interacting protein 2), a protein associated with Huntington's disease, was first discovered in a yeast two-hybrid screen who interacts with huntingtin (3). It is also named as E2-25K, a ubiquitinconjugating (E2) enzyme, and has been shown to be involved in the mediation of amyloid- $\beta$ neurotoxicity and inhibition of proteasome in vitro (4). It functions as a part of machinery of the ubiquitin/proteasome system (UPS). The UPS is involved in a variety of biological pathways. For example, it regulates the cell cycle and modulates the degradation of short-lived and regulatory proteins (5-7). It has been reported that Hip2 can regulate the stability of cyclin B1, a apoptotic and cell cycle regulator, by promoting 
its degradation (8). However, the function of HIP2 in GC remains largely unclear.

In the current study, we analyzed the expression of Hip2 in GC cell lines and patients, and illuminated the role of Hip2 in GC progression. The results showed that the expression of Hip2 was high in human GC and positively correlated with clinical pathology, indicating that Hip2 contributes to high malignancy of GC and poor prognosis. Knockdown of Hip2 significantly inhibited the proliferation, migration and invasion of GC cells in vitro, and reduced tumorigenesis in vivo.

\section{Methods}

\section{Patients and tumor specimens}

Between Jan. 1, 2006 and Dec. 31, 2007, a total of 76 patients (48 males and 28 females) diagnosed with gastric adenocarcinoma registered for the current study. The patients ranged from 30 to 81 years old (median age $=64$ ) and were all hospitalized at the Department of General Surgery, No. 9 People's Hospital, Shanghai Jiao Tong University School of Medicine. Among the patients, 9 experienced total gastrectomy, 57 underwent distal subtotal gastrectomy, and 10 had proximal subtotal gastrectomy. In addition, $\mathrm{D}_{1}$ lymphadenectomy, $\mathrm{D}_{2}$ lymphadenectomy, and $D_{3}$ lymphadenectomy was performed on another 2, 68, and 6 patients, respectively. Postoperative pathological examination revealed 13 cases of early adenocarcinoma, 3 cases of fungal adenocarcinoma, 10 cases of ulcerative adenocarcinoma, 41 cases of invasive ulcerated adenocarcinoma, and 9 cases of diffuse invasive adenocarcinoma. Patient follow-up ended on Dec. 31, 2017, with a median follow-up of 30 months. During this period, 45 patients died of recurrence or metastasis and 16 patients died of other diseases. The survival time ranged from 3 to 130 months.

Tumor tissues after radical surgery were embedded in paraffin and stained with Hip2. The relationships between Hip2 expression and clinicopathological characteristics, survival time after surgery were analyzed respectively. Furthermore, another 20 patients of chronic gastritis (GS) and 20 patients of gastric ulcer (GU) diagnosed by gastroscopic biopsies were enrolled as negative control.

All patients with GC in this study underwent curative gastrectomy, and none of them received preoperative chemotherapy or radiation therapy. A preoperative informed consent was signed by each participating patient according to institutional guidance. All clinicopathological profiles were assessed according to the $8^{\text {th }}$ edition criteria of the American Joint Committee on Cancer (AJCC) (9) and ESMO Clinical Practice Guidelines (10). Prior to the study, approval was obtained from the hospital Review Board and the Ethics Committee of the Medical Faculty at the Shanghai Jiao Tong University.

\section{Immunobistochemistry (IHC) staining}

Four $\mu \mathrm{m}$ serial tissue sections were prepared and then deparaffinized, rehydrated with graded ethanol. Three percent hydrogen peroxide was utilized to block endogenous peroxidase, and $10 \mathrm{mM}$ citrate buffer ( $\mathrm{pH}$ 6.0) was used to retrieve antigen. Then $5 \%$ goat serum was used to block the tissue sections at room temperature for 1 hour. Hip2 $\mathrm{mAb}$ (1:100, Abcam, UK) was diluted in the blocking solution. The slides were then rinsed and incubated with Hip2 overnight at $4{ }^{\circ} \mathrm{C}$, and then incubated at room temperature for 1 hour with the appropriate biotinylated secondary antibody (1:1,000, Boster, China). After this washing series, avidin/biotinylated HRP were used to incubate the tissues (ABC kit, Santa Cruz Biotechnology, USA) in accordance with the manufacturer's instructions. The slides were immersed in diaminobenzidine $(\mathrm{DAB}) / \mathrm{H}_{2} \mathrm{O}_{2}$ substrate solution until the desired staining intensity was achieved. Following this procedure, the slides were counterstained with hematoxylin. For each patient, two independent pathologists who were blind to the patient's status were invited to evaluate and score all the sections. The evaluation of staining result was scored as previously described $(11,12)$. Then the staining cell scores $(0$ point $=0-5 \%$; 1 point $=6-25 \% ; 2$ points $=26-50 \% ; 3$ points $=$ more than $50 \%)$ and staining intensity scores $(0$ point $=$ negative $; 1$ point $=$ weak intensity; 2 points $=$ moderate intensity; 3 points $=$ strong intensity) were summed to obtain a composite score. A score with $\geq 4$ points meant high positive, a score with $\leq 3$ points and $\geq 2$ indicated low positive, and a score with $\leq 1$ point represented negative.

\section{Bioinformatic analysis of clinical data}

GC dataset was obtained from The Cancer Genome Atlas (TCGA) database. Analysis of Hip2 expression was performed using GEPIA (Gene Expression Profiling Interactive Analysis) (http://gepia.cancer-pku.cn/). Overall survival of patients with stomach adenocarcinoma (STAD) 
was assessed by KM plotter: Kaplan-Meier Plotter (http:// kmplot.com/analysis/).

\section{Cell lines}

The human GC cell lines AGS, BGC823, MCG803, MKN28, MKN45, and SGC7901, along with the human normal gastric cell lines GES-1, were purchased from American Type Culture Collection (ATCC, USA). In accordance with the manufacturer's instructions, all the cells were cultured in RPMI-1640 (HyClone) supplemented with $10 \%$ fetal bovine serum (Gibco). All cell lines were kept in a humid atmosphere of $37^{\circ} \mathrm{C}$ with $5 \% \mathrm{CO}_{2}$.

\section{Establishment of stable Hip2 knockdown cell lines}

A scrambled sequence and Hip2 targeting sequence were constructed into the lentiviral expression vector pLKO.1 to obtain control and lenti-shHip2 plasmid. The scrambled sequence is 5'-UUCUCCGAACGUGUCACGUTT-3', and Hip2 targeting sequence is $5{ }^{\prime}$-GGUCCGGUUUAU CACUAAATT-3'. Recombinant lentiviruses expressing corresponding Hip2-shRNA were subsequently produced via co-transfection of lenti-shHip2, helper plasmid psPAX2 and VSV G expressing plasmid pMD2.G on 293T cells with Lipofier (HanBio, Shanghai, China). Forty-eight hours after the transfection, the cultured supernatant was collected, filtered, and concentrated by ultracentrifugation. The viruses were used to infect MKN45 and AGS cell lines to establish stable Hip2-knockdown cell lines. Seventytwo hours after the infection, the cells were treated with $1 \mu \mathrm{g} / \mathrm{mL}$ puromycin. Clones resistant to puromycin were selected and expanded for use in subsequent experiments.

\section{Real time-qPCR}

In accordance with the manufacturer's instructions, TRIzol reagent (Invitrogen, Carlsbad, CA, USA) was used to extract total RNA from GC cells. A RevertAid ${ }^{\mathrm{TM}}$ First Strand cDNA Synthesis kit (Takara) was utilized to conduct reverse transcription, and a QuantiTect SYBR Green kit (Biotool, Houston, TX, USA) was used to perform qPCR. The relative expression levels of mRNA were first normalized to the expressional levels of GADPH, after which they were determined via the comparative Ct-method. For the analysis of gene expression levels, each gene was subjected to the analysis three times. The primer sequences for Hip2 were 5'-GTTCCGTCACAGGGGCTATTT-3' (forward) and 5'-AATACCGTGCGGAGAGTCATT-3' (reverse).

\section{Western blot}

Lysis buffer radioimmunoprecipitation assay (RIPA) (Beyotime Institute of Biotechnology, China) containing protease inhibitors phenylmethanesulfonyl fluoride (PMSF) (Beyotime Institute of Biotechnology, China) was used to dissolve the cells and tissues. Bicinchoninic acid (BCA) protein assay kit (Beyotime Institute of Biotechnology, China) was utilized to determine the protein concentration. Equal amounts of protein $(20 \mu \mathrm{g})$ in $10 \mu \mathrm{L}$ volume were separated on sodium dodecyl sulfate-polyacrylamide gel electrophoresis (SDS-PAGE) and transferred to polyvinylidene difluoride (PVDF) membranes (Millipore, USA). The cell membranes were blocked with $5 \%$ non-fat milk and then incubated with primary antibodies overnight at $4{ }^{\circ} \mathrm{C}$. Incubation with secondary antibodies was performed at room temperature for $1 \mathrm{~h}$. To visualize the protein bands, the enhanced chemiluminescence detection kit (Thermo scientific, USA) was used. The primary antibodies were: Hip2 mAb (1:2,000, Abcam, UK), and GAPDH (1:5,000, Bioworld Technology, USA).

\section{Cell viability assay}

To detect cell growth, cells were seeded at a density of $3 \times 10^{3}$ cells per well in 96 -well plates and grown for a specified period of time. At the end of the period, $10 \mu \mathrm{L}$ of cell counting kit-8 (CCK-8, Dojindo, Japan) solution was added to each well, where the cells were incubated at $37{ }^{\circ} \mathrm{C}$ for another $80 \mathrm{~min}$. Absorbance readings were measured using a $450 \mathrm{~nm}$ plate reader. The impact of background absorbance was eliminated to obtain cell viability.

\section{Cell proliferation assay}

To perform BrdU staining, cells were first immersed in $\operatorname{BrdU}(1 \mathrm{mg} / \mathrm{mL})$ for $4 \mathrm{~h}$, and then fixed with acid ethanol $\left(90 \%\right.$ ethanol, $5 \%$ acetic acid, $\left.5 \% \mathrm{H}_{2} \mathrm{O}\right)$ at room temperature for $30 \mathrm{~min}$. Subsequently, cells were rinsed once with PBS, submerged in $2 \mathrm{M} \mathrm{HCl}$ for $20 \mathrm{~min}, 0.1 \mathrm{M}$ sodium borate, $\mathrm{pH} 8.5$ for $2 \mathrm{~min}$ and rinsed once with PBS. At room temperature, cells were blocked for 1 hour in $10 \%$ BSA in PBS and immersed in anti-BrdU (BD Pharmingen, $1: 500$ in $5 \% \mathrm{BSA}$ in $0.1 \%$ PBS Tween) at $4{ }^{\circ} \mathrm{C}$. Cells were 
rinsed, submerged in anti-mouse Alexa-594 (1:500) at room temperature for $1 \mathrm{~h}$, washed, co-stained with DAPI and mounted.

\section{Cell cycle analysis}

Different types of single cell suspension were prepared and fixed in $70 \%$ ethanol at $4{ }^{\circ} \mathrm{C}$ for $4 \mathrm{~h}$. They were then subjected to treatment with $10 \mathrm{mg} / \mathrm{mL}$ RNase at room temperature for $30 \mathrm{~min}$. After $10 \mu \mathrm{L}$ of propidium iodide (PI, $5 \mathrm{mg} / \mathrm{mL}$ ) was added, the samples were incubated at room temperature for $10 \mathrm{~min}$ and analyzed with a FACS (BD Bioscience Mountain View, CA, USA).

\section{Wound-healing assay}

Cells were cultured at $80-90 \%$ confluence in six-well plates. Ten- $\mu \mathrm{L}$ plastic tips were used to scrape the wound areas to form a straight line. Culture plates were washed three times to remove cell debris and changed to the serumfree medium. An inverted research microscope eclipse TS100/100-F (Nikon, Japan) was used to monitor the healing process by taking images every $24 \mathrm{~h}$. The healing rate $=(0-48 \mathrm{~h}$ width $) / 0 \mathrm{~h}$ width .

\section{Transwell migration and invasion}

To perform migration assay, a 24-well 8 - $\mu$ m pore size transwell chamber (Corning, USA) was used. The bottom of the transwell chamber was coated with BD Matrigel ${ }^{\mathrm{TM}}$ Matrix Basement Membrane (BD Biosciences, USA) to measure cell invasion. $1 \times 10^{5}$ cells were immersed in $200 \mu \mathrm{L}$ serum free medium in the upper chamber, while the lower chamber was filled with $600 \mu \mathrm{L}$ medium containing $20 \%$ fetal bovine serum as a chemoattractant. The incubation process lasted for $48 \mathrm{~h}$, after which the non-invading cells on the upper surface of the membrane were scraped away. Invading cells on the lower side of the membrane were fixed in $4 \%$ paraformaldehyde and stained with $0.05 \%$ crystal violet. A microscope was used to record the number of invasive cells. These experiments were performed in triplicate.

\section{Xenograft tumor}

Male nude athymic BALB/c mice (S, China) of approximately four weeks were obtained from SLAC Laboratory Animal Co., Ltd. (Shanghai, China). The mice were subcutaneously injected with $5 \times 10^{6}$ cells from the shCtrl or shHip2 group in $0.2 \mathrm{~mL}$ volume in their right flanks, respectively. This was followed by measurements of the tumors size every three days. The formula used for the measurement of tumor size was: volume $=$ (length $\times$ width $\left.{ }^{2}\right) / 2$. At the end of the experiment, the mice were killed. Their tumor was then weighted and fixed in $10 \%$ neutral-buffered formalin. All the experimental procedures complied with Shanghai Jiao Tong University guidelines for the use of animals, and were approved by The Animal Care and Utilization Committee of Shanghai Jiao Tong University.

\section{Statistical analysis}

Results are presented as means \pm SD. Chi-square analysis was performed to estimate clinicopathological parameters. The Kaplan-Meier method was used to obtain survival curves, and survival rate was analyzed using the log-rank method. For cell and animal experiments, the data were submitted to one-way ANOVA or Student's $t$ test. All the analyses were performed using Graphpad Prism 8.0 software (Graphpad Software; San Diego, CA, USA), and $\mathrm{P}$ values of $<0.05$ indicated that the results were statistically significant.

\section{Results}

\section{Hip2 expression was elevated in GC cancer cell lines and tissues}

To illuminate the function of Hip2 in human GC progression, we firstly recorded Hip2 expression in GC cell lines (gastric carcinoma cell lines MGC-803 and SGC-7901, and gastric adenocarcinoma cell line BGC-823, MKN28, MKN45 and AGS) and gastric epithelial cell line GES-1. The levels of mRNA (Figure 1A) and protein (Figure 1B) in Hip2 are higher in GC cell lines than those in epithelial cell line. Next, we investigated the expression profile of Hip2 in human GC tissues. The samples comprised 116 patients, including 20 patients with GS, 20 with GU, and 76 with GC. A summary of the patient data is presented in Table 1. The protein levels of Hip2 in GC specimens were examined by immunohistochemical staining. All the specimens were categorized into three groups (negative, low positive and high positive) according to the expression score based on the density and percentage of IHC staining. All GS patients belong to negative group, $15 \%$ (3/20) in patients with 
A

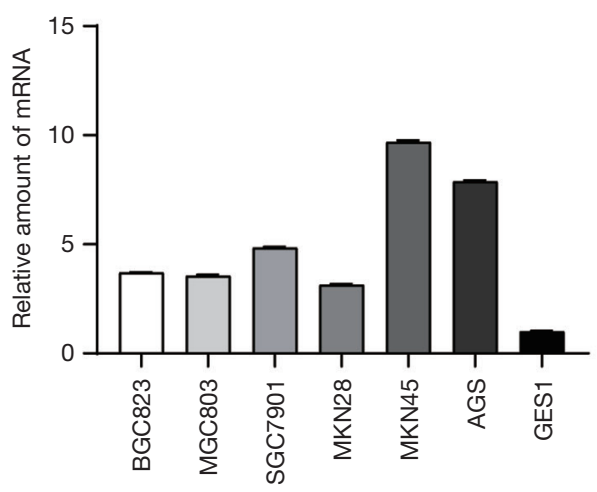

C
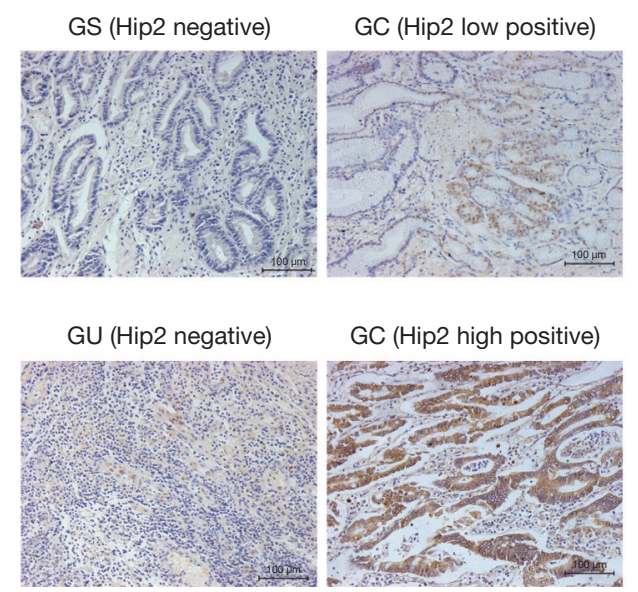

B

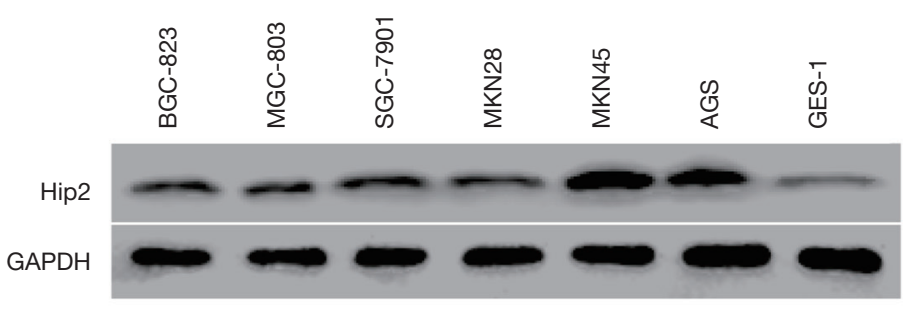

D

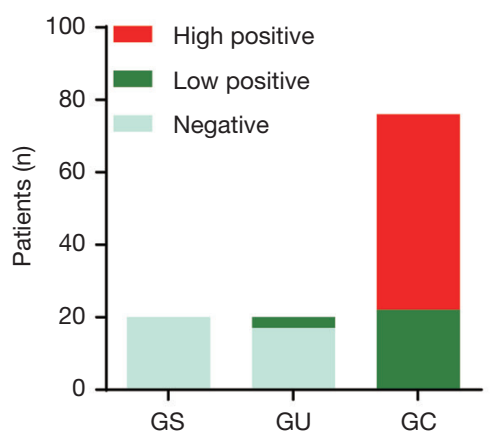

$E$

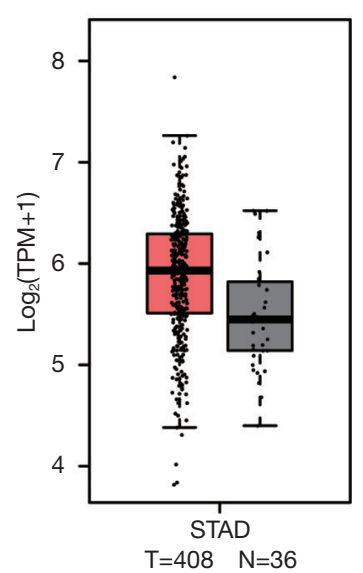

Figure 1 Hip2 expression is elevated in human gastric cancer lines and tissues. (A) The mRNA levels of Hip2 were assessed by RT-qPCR in 6 gastric cancer cell lines and gastric epithelial cells; (B) the protein levels of Hip2 were assessed by Western Blot in 6 gastric cancer cell lines and gastric epithelial cells; (C,D) the expression of Hip2 was assessed by IHC staining in the GS, GU and GC tissue; (E) Hip2 was elevated in human STAD specimens. Analysis of the mRNA levels of Hip2 in normal (N, n=36), and stomach adenocarcinoma (STAD, n=408) from a TCGA database. GS, chronic gastritis; GU, gastric ulcer; GC, gastric cancer.

GU belong to low positive group and the others belong to negative group, and $28.9 \%(22 / 76)$ in patients with GC belong to low positive group and the others belong to high positive group. The expression of HIP2 was $0 \%$ $(0 / 20)$ in patients with GS, $15 \%(3 / 20)$ in patients with GU, and 100\% (76/76) in patients with GC (Figure 1C,D). Furthermore, the data from TCGA shown that the mRNA levels of Hip2 in STAD were remarkably higher than that in normal gastric tissues (Figure 1E). These data indicated that the elevated Hip2 may promote GC progression.

\section{High levels of Hip2 predicted poor prognosis in GC patients}

To explore the correlation between clinicopathological features of GC patients and the levels of their Hip2 expression, we evaluated various key parameters for tumors from patients, including tumor size, lymphatic vessel invasion (LVI), T stage, lymph node metastasis, metastatic lymph nodes ratio, and TNM stage (Table 1). Strikingly, Hip2 expression levels were significantly correlated with tumor size $(\mathrm{P}=0.012)$, LVI $(\mathrm{P}=0.003)$, $\mathrm{T}$ stage $(\mathrm{P} \leq 0.001)$, lymph node metastasis $(\mathrm{P} \leq 0.001)$, metastatic lymph nodes ratio $(\mathrm{P} \leq 0.001)$ and $\mathrm{p} T \mathrm{NM}$ stage $(\mathrm{P} \leq 0.001)$, respectively. But no significant correlation was found for gender $(\mathrm{P}=0.956)$, histology type $(\mathrm{P}=0.346)$ or venous invasion $(\mathrm{P}=0.065)$ (Table 1). The overall survival rate in Hip2 low positive group was higher than that in Hip2 high positive group (Figure $2 A$ ). The five-year and ten-year survival rate 
Table 1 Distribution of clinical characteristics stratified by Hip2 protein expression in 76 patients with GC

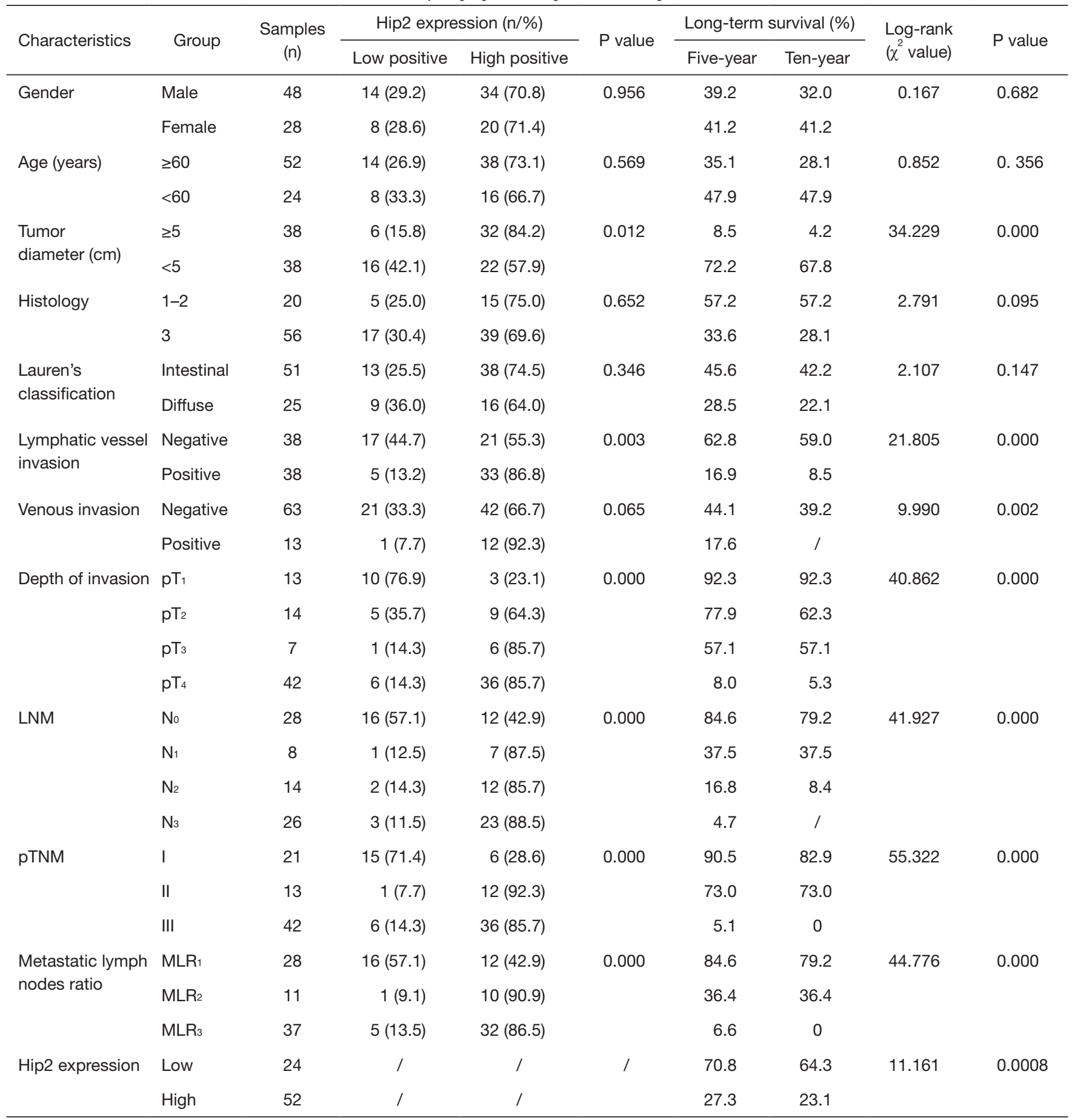

of GC patients in Hip2 high positive group were $27.3 \%$ and $23.1 \%$ respectively, compared with that of $70.8 \%$ and $64.3 \%$ in Hip2 low positive group in GC (Figure $2 A$ and Table 1). The data from TCGA also showed that the overall survival rate was lower in the patients who exhibit high levels of Hip2 expression in STAD (Figure 2B). These results indicate that the patients who expressed high levels of Hip2 predicted poor prognosis. 
A

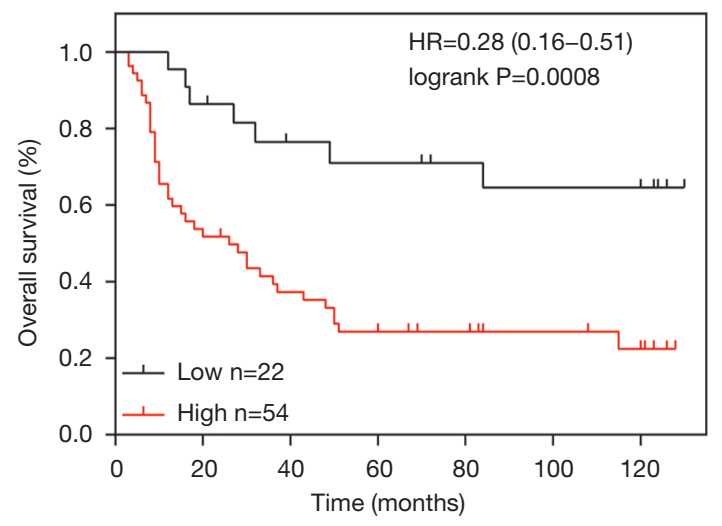

B

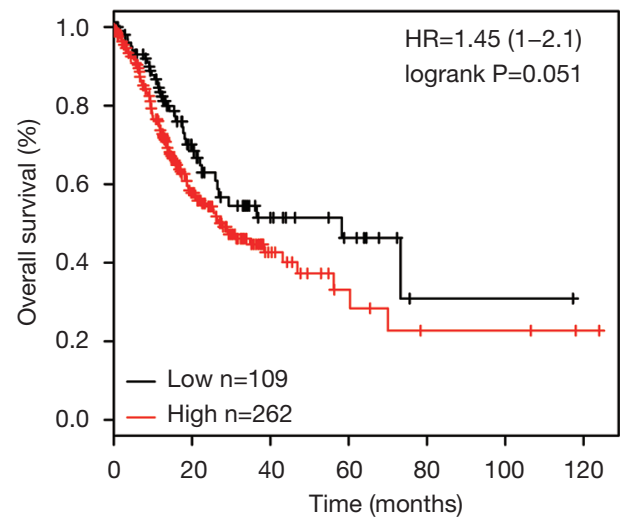

Figure 2 High levels of Hip2 predict poor prognosis. (A) The overall survival rate of GC patients with different expression levels of Hip2. The data was from our enrolled patients. (B) The overall survival rate of STAD patients with different Hip2 expression levels. The data was from TCGA database.

\section{Knockdown of Hip2 inbibited GC cell proliferation}

To clarify the role of Hip2 in GC cell proliferation, short hairpin RNAs against Hip2 was used to knock down Hip2 expression in 2 human GC cell lines, MKN45 and AGS, because Hip2 expression is relatively higher in these two cell lines compared with other GC cell lines. The stable Hip2 knockdown MKN45 and AGS cells were established and used for following assay. Hip2 knockdown and control cells seeded into 96 well plates, and cell growth was subjected to CCK-8 assay. As shown in Figure $3 A$ and $B$, knockdown of Hip2 inhibited GC cell growth in MKN45 (Figure $3 A$ ) and AGS (Figure 3B) cells. Then, BrdU staining was taken to confirm the effect of Hip2 on cell proliferation. Consistently, knockdown of HIP2 resulted in a decreased BrdU positive cells in MKN45 (Figure 3C) and AGS (Figure 3D) cells. Furthermore, cell cycle of Hip2 knockdown and control cells was analyzed by FACS. The results shown that knockdown of Hip2 induced G2/M arrest in MKN45 (Figure 3E) and AGS (Figure 3F) cells. These data indicated that reduction of Hip2 inhibited GC cell proliferation.

\section{Knockdown of Hip2 suppressed GC cell migration and invasion}

As migration and invasion are two key characteristics of cancer cells, we also measured whether knockdown of Hip2 impaired the migration and invasion of GC cells. First, the GC cells were tested for their wound healing ability. As shown in Figure $4 A$ and $B$, knockdown of Hip2 decreased GC cell wound healing in MKN45 (Figure 4A) and AGS (Figure $4 B$ ) cells. Furthermore, transwell migration and invasion assay were performed to detect cell migration and invasion. We found that knockdown of Hip2 reduced the ability of MKN45 and AGS cells to pass through the basement membrane in the presence or absence of matrigel (Figure $4 C, D, E, F)$. The Migration and invasion rates of Hip2 knockdown MKN45 and AGS cells were lower than those control cells (Figure 4C,D,E,F). Knockdown of Hip2 inhibited migration (Figure $4 C$ ) and invasion (Figure $4 E$ ) by about $15 \%$ and $70 \%$, respectively, in MKN45 (Figure 4C,E) cells. In AGS cells, migration (Figure 4D) and invasion (Figure $4 F$ ) were suppressed by about $70 \%$ and $60 \%$, respectively (Figure $4 D, F$ ). These data indicated that reduction of Hip2 inhibited the migration and invasion of GC cells.

\section{Knockdown of Hip2 inbibited MKN45 cell tumorigenesis}

The above results demonstrated that reduction of Hip2 suppressed the proliferation, migration and invasion of GC cells. Then, a xenograft tumor model was constructed to analyze the effects of Hip2 downregulation on GC cell tumorigenesis in vivo. Hip2 knockdown and control MKN45 cells were subcutaneously injected into nude mice. The tumors were harvested at the experimental end point, and the tumor size and weight were measured. As shown in Figure $5 A$ and $B$, the tumors in Hip2 downregulation group 
A

MKN45

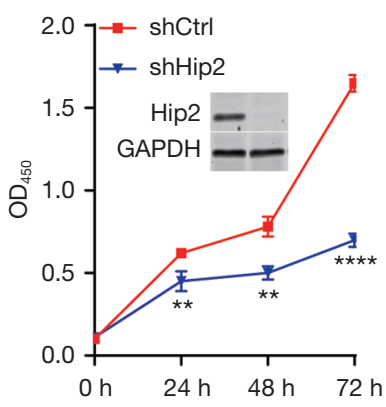

B

AGS

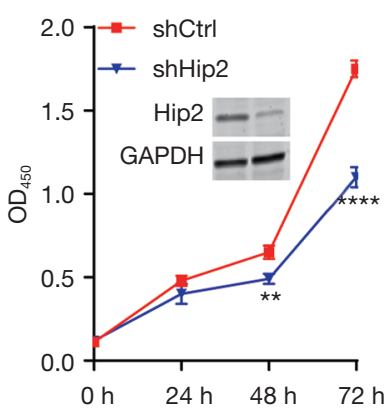

C

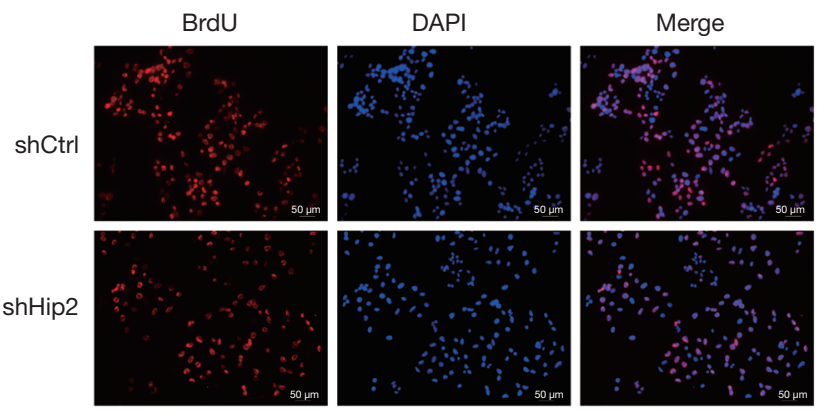

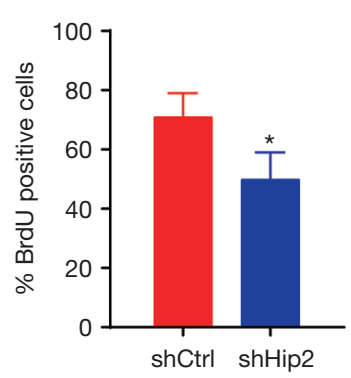

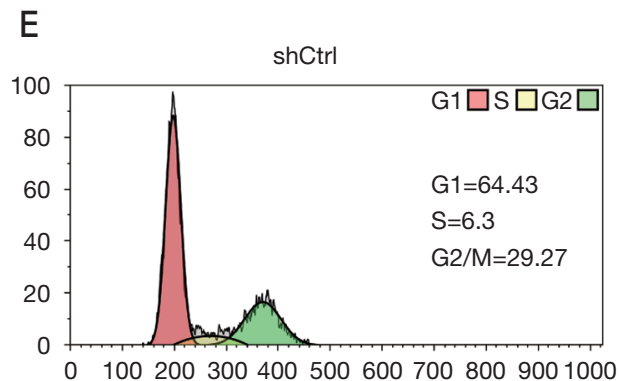

MKN45
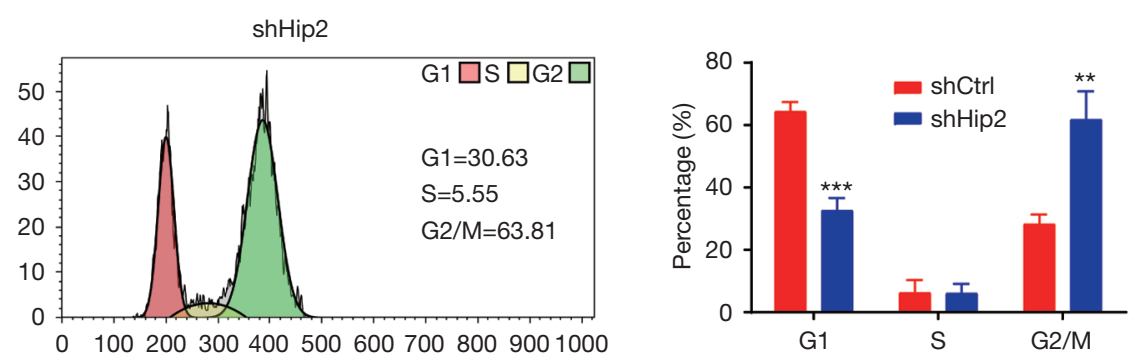

$\mathrm{F}$

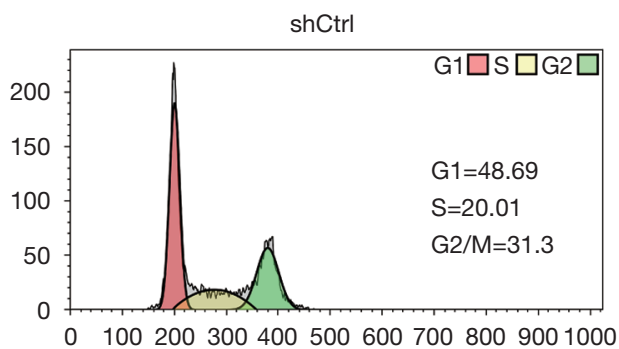

BrdU

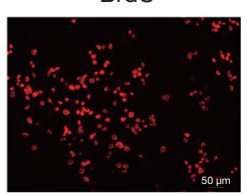

shHip2

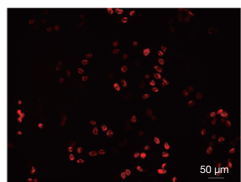

DAPI
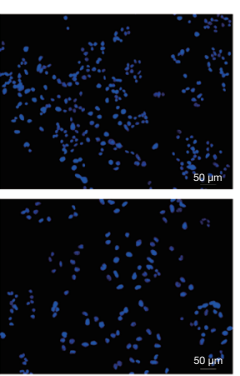

AGS

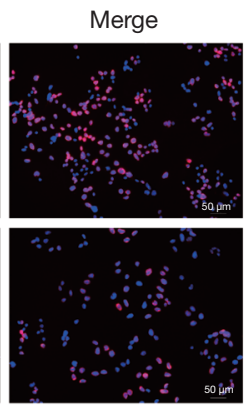

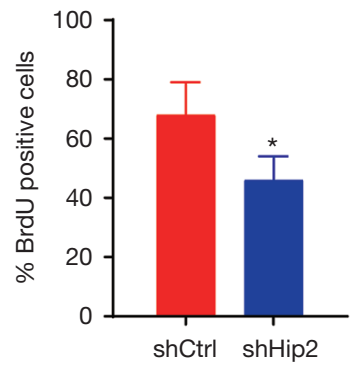

AGS
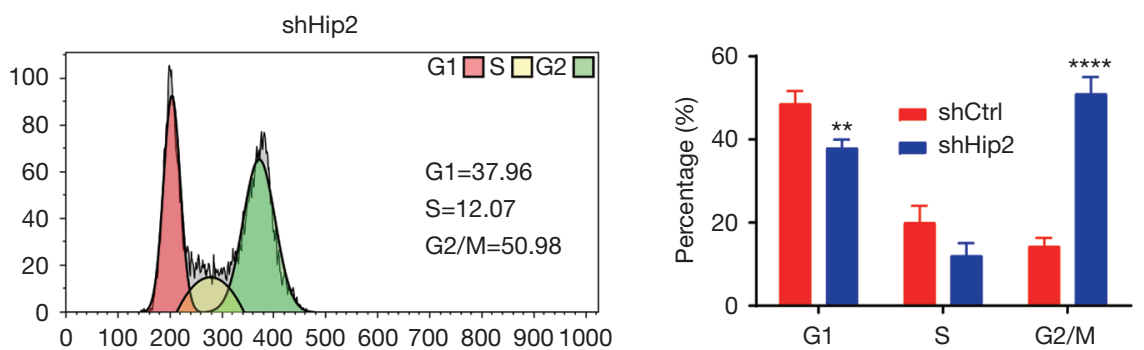

Figure 3 Knockdown of Hip2 suppressed the migration and invasion of GC cells. (A,B) Knockdown of Hip2 suppressed GC cell growth in MKN45 (A) and AGS (B) cells. Cell growth was recorded by CCK-8 assay. (C,D) Knockdown of Hip2 suppressed GC cell replication in MKN45 (C) and AGS (D) cells. Cell replication was performed by BrdU staining. (E,F) Knockdown of Hip2 induced GC cell G2/M arrest in MKN45 (E) and AGS (F) cells. Flow cytometry was used to analyze cell cycle. ${ }^{*}, \mathrm{P}<0.05 ;{ }^{* *}, \mathrm{P}<0.01 ;{ }^{* * *}, \mathrm{P}<0.001 ;{ }^{* * * *}, \mathrm{P}<0.0001$. 

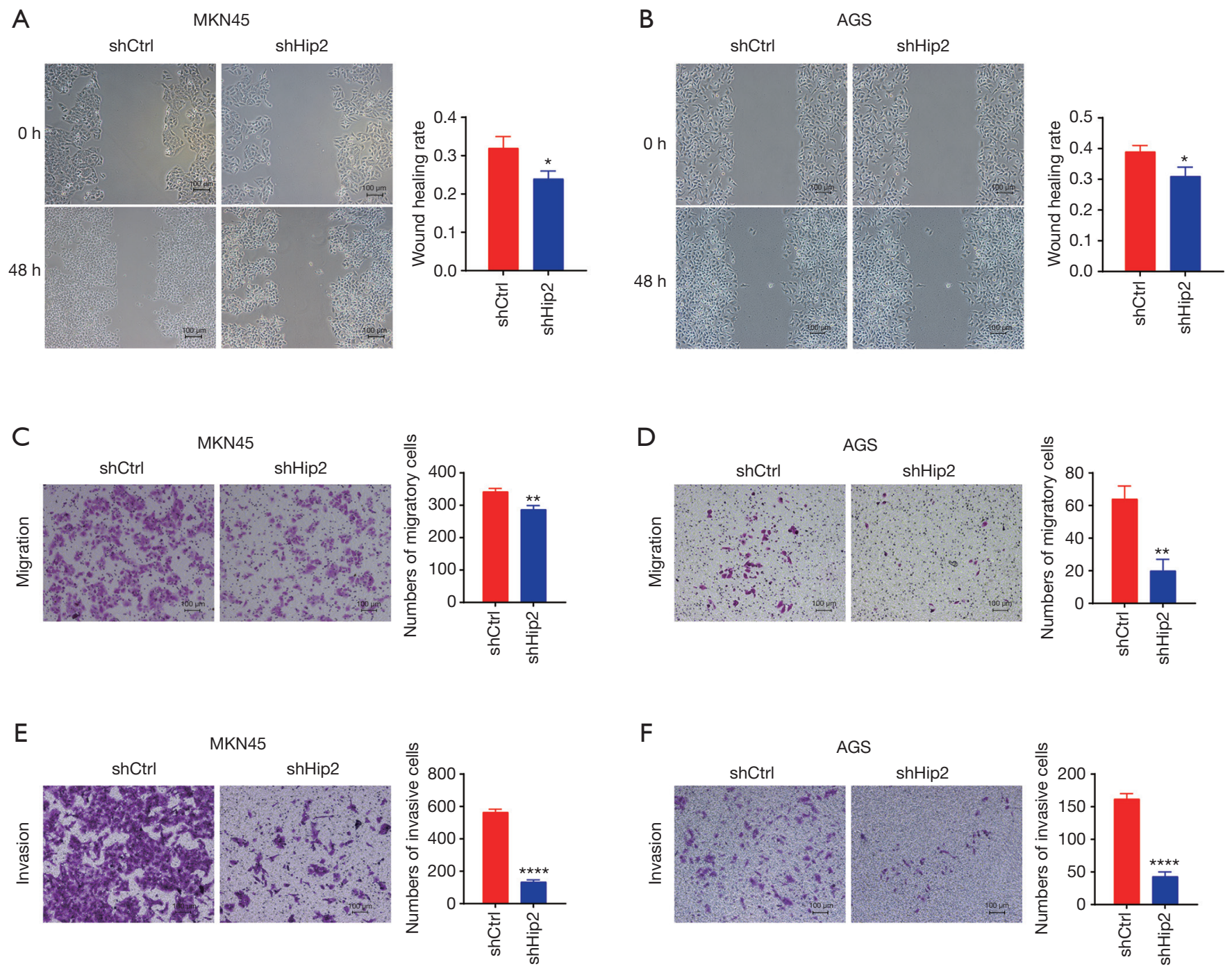

Figure 4 Knockdown of Hip2 suppressed GC cell migration and invasion. (A,B) Knockdown of Hip2 suppressed GC cell wound healing in MKN45 (A) and AGS (B) cells; (C,D) knockdown of Hip2 suppressed GC cell migration in MKN45 (C) and AGS (D) cells; (E,F) knockdown of Hip2 suppressed GC cell invasion in MKN45 (E) and AGS (F) cells. *, $\mathrm{P}<0.05$; **, $\mathrm{P}<0.01$; ****, $\mathrm{P}<0.0001$.

grew much slower and weighed much less compared with control group. The tumor volume was $1,261.0 \pm 224.9 \mathrm{~mm}^{3}$ in the shCtrl group and $369.2 \pm 150.0 \mathrm{~mm}^{3}$ in the shHip2 group, the tumor weight was $0.5619 \pm 0.0635 \mathrm{~g}$ in the shCtrl group and $0.1797 \pm 0.0449 \mathrm{~g}$ in the shHip2 group, respectively. There were less inflammatory cells infiltrated in shHip2 group tumor compared to shCtrl group (Figure 5C). As expected, expression of Hip2 performed by IHC went an apparent decline in the xenograft tumors of shHip2 group (Figure 5D). These data indicated that reduction of Hip2 suppressed tumorigenesis.

\section{Discussion}

In the present work, we clearly demonstrate the key role of Hip2 in GC progression: (I) a obviously up-regulation of Hip2 in GC was identified by analyzing the data which obtained from our enrolled specimens and TCGA dataset; (II) knockdown of Hip2 significantly reduced the proliferation, migration and invasion of GC cells; and (III) knockdown of Hip2 suppressed GC tumorigenesis.

Hip2, a human homolog of the Ubc1 enzyme of $S$. cerevisiae, is a ubiquitin-conjugating enzyme encoded 
A

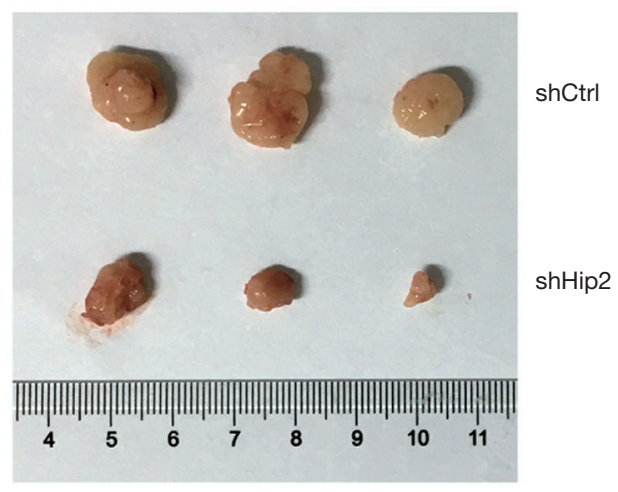

C

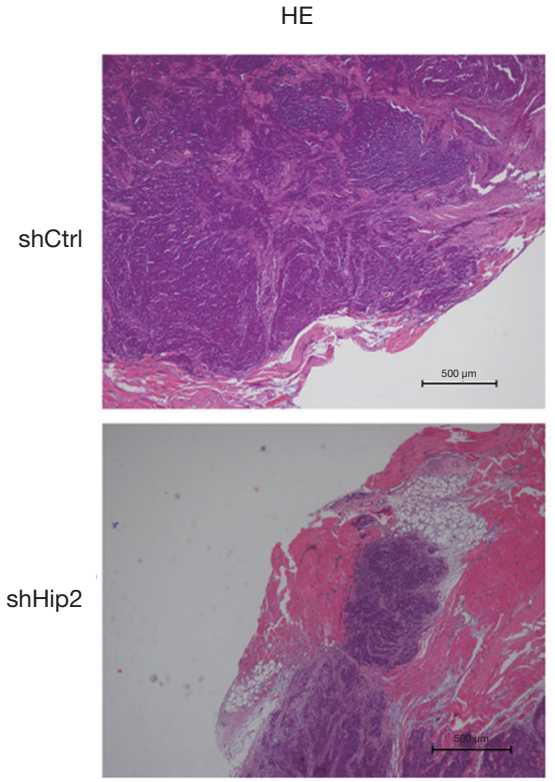

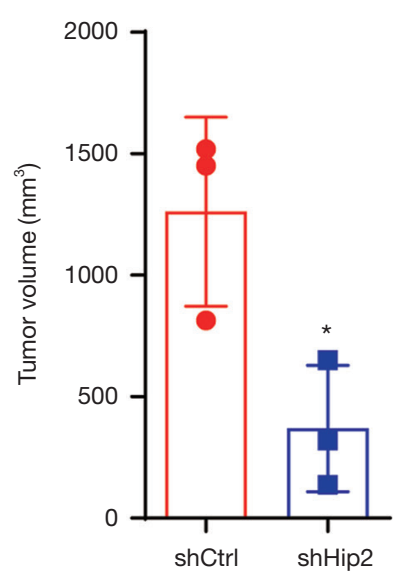

B

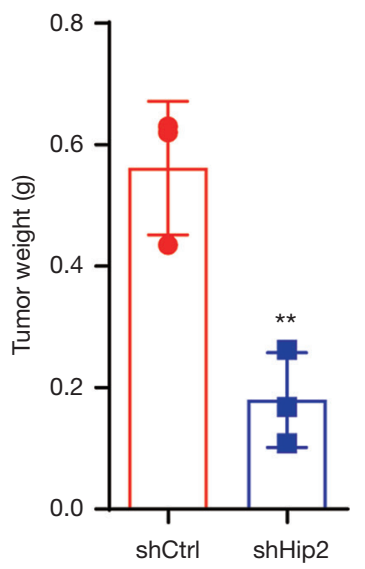

D
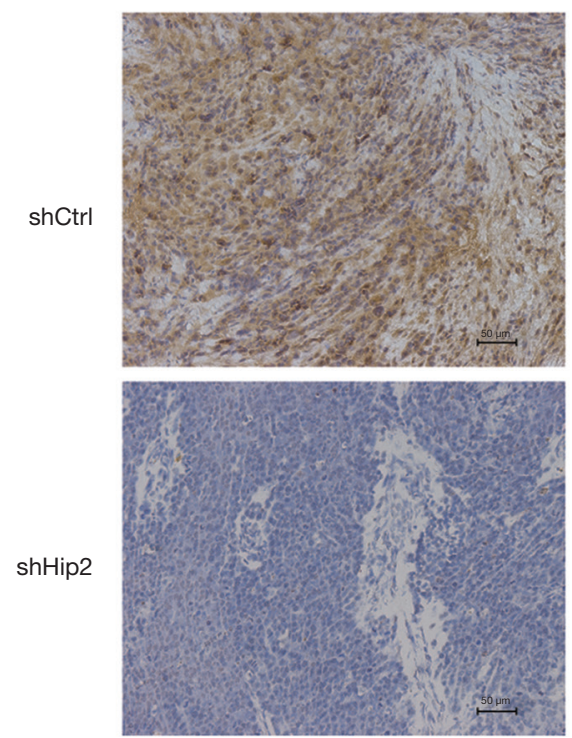

Figure 5 Knockdown of Hip2 inhibited MKN45 GC cells tumorigenesis. (A,B) The size and weight of subcutaneous tumor in shCtrl and shHip2 group; (C) the HE staining of xenograft tumors in shCtrl and shHip2 group; (D) the expression of Hip2 in tumors sections of shCtrl and shHip2 group were determined by IHC. * $\mathrm{P}<0.05 ;{ }^{* *}, \mathrm{P}<0.01$.

by the $U B E 2 K$ gene (13). Besides acting as an E2 conjugating enzyme, HIP2 also has basal E3-independent polyubiquitination activity, which is involved in UPSmediated protein degradation $(14,15)$. Protein degradation by the UPS is carried out through two successive steps: ubiquitination and degradation. Ubiquitination is achieved by a cascade of enzymes that activate (E1), conjugate
(E2) and ligate (E3) ubiquitin to target proteins. In the ubiquitination of proteins to be degraded, E2 proteins play an integral role by interacting specifically with a subset of E3 and targeting proteins. Furthermore, Hip2 is a special type of E2 because it not only functions as the E2 conjugating enzyme, but also has UBA domains that produce an unanchored Lys48-polyubiquitin chain and 
ubiquitinate substrates without ubiquitin ligases $(14,15)$. Previously, Bae et al. reported that Hip2 promoted cyclin B1 degradation through the ubiquitin proteasome pathway (8). Cyclin B1 is artificially controlled, and helpful for mitotic exit and completion of the cell cycle. We speculate that Hip2 might be involved in the mediation of cell cycle and the regulation of cell proliferation. As the results of this study, Hip was elevated in GC and suppressed GC cell growth in vitro (Figure 3) and in vivo (Figure 5). Migration and invasion are two key characteristics of cancer cells. The protein which can control cancer cell migration and invasion are useful therapies in the treatment of cancer. As the data shown in Figure 4 suggest, we also demonstrated that knockdown of Hip2 impaired GC cell migration and invasion.

\section{Conclusions}

This study confirmed that Hip2, a ubiquitin-conjugating enzyme, was elevated in GC cells and patients. Reduction of Hip2 suppressed cell proliferation, cell cycle, wound healing, cell migration and invasion in GC cells. In this study, it was found that elevated Hip2 could promote GC progression. Therefore, Hip2 holds promise as a potential target for the treatment of GC.

\section{Acknowledgments}

Funding: This work was supported by grants of National Natural Science Foundation of China (81470792), Shanghai Shenkang Hospital Development Center (16CR4010A), and Municipal Health and Family Planning Commission of Shanghai (20164Y0255).

\section{Footnote}

Conflicts of Interest: All authors have completed the ICMJE uniform disclosure form (available at http://dx.doi. org/10.21037/tcr.2019.12.12). The authors have no conflicts of interest to declare.

Ethical Statement: The authors are accountable for all aspects of the work in ensuring that questions related to the accuracy or integrity of any part of the work are appropriately investigated and resolved. The study was conducted in accordance with the Declaration of Helsinki (as revised in 2013). All the patients selected for our study were fully informed about our experimental protocols, which were approved by the Ethics Committee of the Medical Faculty at the Shanghai Jiao Tong University. All the animal experimental procedures complied with Shanghai Jiao Tong University guidelines for the use of animals, and were approved by The Animal Care and Utilization Committee of Shanghai Jiao Tong University.

Open Access Statement: This is an Open Access article distributed in accordance with the Creative Commons Attribution-NonCommercial-NoDerivs 4.0 International License (CC BY-NC-ND 4.0), which permits the noncommercial replication and distribution of the article with the strict proviso that no changes or edits are made and the original work is properly cited (including links to both the formal publication through the relevant DOI and the license). See: https://creativecommons.org/licenses/by-nc$\mathrm{nd} / 4.0 /$.

\section{References}

1. Bray F, Ferlay J, Soerjomataram I, et al. Global cancer statistics 2018: GLOBOCAN estimates of incidence and mortality worldwide for 36 cancers in 185 countries. CA Cancer J Clin 2018;68:394-424.

2. Chen $W$, Zheng R, Baade PD, et al. Cancer statistics in china, 2015. CA Cancer J Clin 2016;66:115-32.

3. Kalchman MA, Graham RK, Xia G, et al. Huntingtin is ubiquitinated and interacts with a specific ubiquitinconjugating enzyme. J Biol Chem 1996;271:19385-94.

4. Song S, Kim SY, Hong YM, et al. Essential role of e2-25k/ hip-2 in mediating amyloid-beta neurotoxicity. Mol Cell 2003;12:553-63.

5. Rubinsztein DC. The roles of intracellular proteindegradation pathways in neurodegeneration. Nature 2006;443:780-6.

6. Rousseau A, Bertolotti A. Regulation of proteasome assembly and activity in health and disease. Nat Rev Mol Cell Biol 2018;19:697-712.

7. Qi J and Ronai ZA. Dysregulation of ubiquitin ligases in cancer. Drug Resist Updat 2015;23:1-11.

8. Bae Y, Choi D, Rhim H, et al. Hip2 interacts with cyclin b1 and promotes its degradation through the ubiquitin proteasome pathway. FEBS Lett 2010;584:4505-10.

9. Amin MB, Greene FL, Edge SB, et al. The eighth edition AJCC cancer staging manual: Continuing to build a bridge from a population-based to a more "personalized" approach to cancer staging. CA Cancer J Clin 2017;67:93-9. 
10. Smyth EC, Verheij M, Allum W, et al. Gastric cancer: ESMO clinical practice guidelines for diagnosis, treatment and follow-up. Ann Oncol 2016;27:v38-v49.

11. Chen DH, Yu JW, Wu JG, et al. Significances of contactin-1 expression in human gastric cancer and knockdown of contactin-1 expression inhibits invasion and metastasis of mkn45 gastric cancer cells. J Cancer Res Clin Oncol 2015;141:2109-20.

12. Du Y, Jiang B, Song S, et al. Metadherin regulates actin cytoskeletal remodeling and enhances human gastric cancer metastasis via epithelial-mesenchymal transition. Int J Oncol 2017;51:63-74.

Cite this article as: $\mathrm{Wu} \mathrm{J}$, Tian B, Yang J, Huo H, Song Z, Yu J, Gu Y. Reduction of Hip2 suppresses gastric cancer cell proliferation, migration, invasion and tumorigenesis. Transl Cancer Res 2020;9(2):774-785. doi: 10.21037/tcr.2019.12.12
13. Chen ZJ, Niles EG and Pickart CM. Isolation of a cDNA encoding a mammalian multiubiquitinating enzyme (e225k) and overexpression of the functional enzyme in escherichia coli. J Biol Chem 1991;266:15698-704.

14. Oh KJ, Kalinina A and Bagchi S. Destabilization of RB by human papillomavirus e7 is cell cycle dependent: E2-25k is involved in the proteolysis. Virology 2010;396:118-24.

15. Cook BW, Barber KR, Shilton BH, et al. The hip2 ubiquitin conjugate forms a non-compact monomeric thioester during di-ubiquitin synthesis. PLoS One 2015;10:e0120318. 